\title{
Measuring functional outcome after total hip replacement with subject-specific hip joint loading
}

Proc IMechE Part $\mathrm{H}$ :

$\mathrm{J}$ Engineering in Medicine

226(I2) 939-946

(c) IMechE 2012

Reprints and permissions:

sagepub.co.uk/journalsPermissions.nav DOI: $10.1177 / 09544$ I 1912447728

pih.sagepub.com

(SAGE

\author{
Tim Weber ${ }^{1,2}$, Sebastian Dendorfer², Silvia Dullien', Joachim Grifka', \\ Gijsbertus Jacob Verkerke ${ }^{3,4}$ and Tobias Renkawitz'
}

\begin{abstract}
Total hip replacement is an often-performed orthopedic surgical procedure; the amount of procedures undertaken will increase since our life expectancy is growing. In order to optimize function, hip biomechanics should be restored to as near normal as possible. The goal of this pilot study was to determine whether or not it is feasible to compute the vectorial hip reaction force pathways on the head of the prosthesis and the force angles relative to the cup of the prosthesis that occur during gait in total hip replacement patients, serving as an objective measurement of the functional outcome following hip replacement.

A three-dimensional gait analysis, measuring ground reaction forces and kinematics, was performed. The data retrieved from the gait analysis was used as the input for the musculoskeletal model to compute vectorial joint reaction forces for data processing. To evaluate the position and orientation of the joint reaction forces, the force path, as well as the force angles for the operated and non-operated joint, has been calculated during the stance phase of the specific leg.

The force path for subject 2 on the non-operated side is only located in the posterior-lateral quarter, as is the force path for subject I. In contrast to this subject, the force path for subject 2 at the operated hip joint can be found only within the anterior quarter of the head of the implant, where it is nearly equally distributed in the medio-lateral half of the prosthesis head. The force-inclination angles on the cup of subject I, with respect to the plane of the socket face, indicates that the force vector is mainly positioned in the same quadrant when compared with subject 2 (in a cup-fixed coordinate system). The force-anteversion angle behaves similarly to the force-inclination angle, even when the effects are not as pronounced.

The proposed methods in this article are aiming to define two functional outcomes of total hip replacement that are related to wear and rim loading. It is accepted that wear is not only a function of time, but a function of use. Owing to the methods listed in this article, we are able to determine a) the applied force and b) the sliding distance (force pathway) in a subject-specific manner. The computed hip-reaction force angles and the distance to the rim cup are a measurement for cup or rim loading, and occurs in the so-called safe-zones. This method may well give us insight into the biomechanical situation during gait, after receiving total hip replacement, that we need to fully understand the mechanisms acting on a hip joint and to prove a possible increase of functional outcome after receiving total hip replacement.
\end{abstract}

\section{Keywords}

Total hip replacement, subject-specific musculoskeletal modeling, functional outcome, force pathways, force angles, critical hip joint loading, rim loading, cup loading

Date received: 10 January 2012; accepted: 10 April 2012

\section{Introduction}

Total hip replacement (THR) is an often-performed orthopedic surgical procedure; the amount of procedures undertaken will increase since our life expectancy is growing. Pain relief and restoration of hip biomechanics are the desired goals in THR. ${ }^{1}$ In order to optimize function, hip biomechanics should be restored to as near normal as possible.

\footnotetext{
'Department of Orthopedic Surgery, Regensburg University Medical Center, Germany

${ }^{2}$ Laboratory of Biomechanics, University of Applied Science in Regensburg, Germany

${ }^{3}$ Department of Biomedical Engineering, University Medical Center Groningen, The Netherlands

${ }^{4}$ Department of Biomechanical Engineering, University of Twente, The Netherlands
}

\section{Corresponding author:}

Tim Weber, Department of Orthopedic Surgery, Regensburg University Medical Center, Laboratory for Gait- and Motion Analysis, Kaiser Karl V. Allee 3, Bad Abbach 93077, Germany.

Email: Tim.Weber@klinik.uni-regensburg.de 
To assess the functional status and post-operative outcome of THR, gait analysis using body-fixed sensors was introduced several years ago. ${ }^{2,3}$ Numerous studies have proven that gait analysis enables objective measurement of subject-specific differences following THR and to determine the functional outcome. ${ }^{4-8}$ However, these studies mainly focus on kinematics and temporospatial parameters as well as the measurement of ground reaction forces. Hip reaction forces cannot be measured, however, these are important because detailed knowledge about the in-vivo load case of the hip can be used, not only for further implant improvement, but also as a measure for the post-operative outcome after an operation. ${ }^{9,10}$ Recently, novel simulation techniques, like musculoskeletal modeling (MM), have been established for research of patient-specific biomechanics ${ }^{1-15}$ and they have been proven as valid and meaningful in various studies. ${ }^{16,17}$ These models are capable of computing the patient-specific hip reaction forces by means of subjectspecific data, like motion capture marker trajectories and ground reaction force measurements retrieved from gait analysis. Based on the marker trajectories the models are scaled to the subject-specific anatomy, this information is used in order to optimize the subject-specific kinematics, ${ }^{18}$ not to mention the highly detailed and realistic modeling of the muscles. ${ }^{19}$ The use of an inverse dynamics approach also makes the models very efficient from a computational point of view. By means of a properly defined patient collective, ${ }^{20}$ a large cohort of these models can easily be employed and will allow us to make quantitative statements about the hip biomechanics following THR.

Only few biomechanical studies have been conducted on critical hip joint loading. To the authors knowledge only Nicholas et al. ${ }^{21}$ has conducted critical hip joint loading experiments using a test rig to compare different prosthesis types. Scifert et al. $^{22}$ is one of the few groups that has developed finite element models aiming at hip joint failure and conducted validation experiments using a similar test rig as Nicholas et al. Its results indicate a biomechanical mechanism dealing with the resisting moment in a hip joint, which is mainly dependent on the distance of the resulting joint reaction force to the rim of the cup and its magnitude. Both of the aforementioned studies ${ }^{21,22}$ focus their research on implant-on-implant impingement. Stewart et al. ${ }^{23}$ is the only group, to our knowledge, that includes soft tissue impingement by implementing a capsule representation in a total hip joint finite element model. They also propose that impingement is not the only cause of hip joint failure, but also shearing and sliding-out processes might be involved. The work of Saikko et al. and Calonius et al. ${ }^{24-26}$ describes a technique using hip joint simulators to measure the slide tracks on a hip implant while walking, thereby providing an interesting tool to prove possible sliding-out mechanisms with respect to the hip joint kinematics and kinetics. Nadzadi et al. ${ }^{27}$ analyzed different movement patterns by means of motion capture (MoCap) data and a computational model aiming to predict any hip joint failure within the patient. They also assume that the critical hip joint loading is highly sensitive to vectorial joint contact forces. Taken together, some biomechanical principles of critical hip joint loading after THR are indicated, but remain to be proven and quantified. Especially the vectorial pathways of the hip reaction forces seem to offer a promising approach in order to clarify the effects.

The goal of this pilot study was to determine whether or not it is feasible to compute the vectorial hip reaction force pathways on the head of the prosthesis and the force angles relative to the cup of the prosthesis that occur during gait in THR patients. The methods proposed in this article are aimed at defining two functional outcomes of THR that are related to wear (force pathways) and rim loading (force angles).

\section{Materials and methods}

Between 2007 and 2009 patients underwent THR using the minimally invasive single-incision Micro-Hip ${ }^{\circledR}$ approach in a lateral-decubitus position at the Orthopedics Department, Regensburg University Medical Center, Germany. Press-fit acetabular components and cement-free hydroxyapatite-coated stems (Pinnacle cup, Corail stem, DePuy, Warsaw, IN, USA) with $32 \mathrm{~mm}$ metal heads were used. Post-operatively, a pelvic/femoral computed tomography (CT) scan was performed. The three-dimensional (3D) position with respect to the femoral coordinate system and the individual orientation (radiographic inclination and anteversion) of the acetabular component was evaluated by an independent external institute (MeVis,Bremen, Germany) on a $3 \mathrm{D}$ reconstruction of the pelvis using image-processing software (based on MeVisLab, MeVis). The post-operative definition of the acetabular planes for cup inclination and anteversion was based upon the radiographic plane and coordinate system according to Murray et al. ${ }^{28}$ For this pilot study, one subject with a high cup inclination (subject 1; inclination: $48.2^{\circ}$; anteversion: $19.6^{\circ}$ ) and one with a high cup anteversion (subject 2; inclination: $38.7^{\circ}$; anteversion: $33.3^{\circ}$ ) were selected for further analysis. A 3D gait analysis, measuring ground reaction forces (three degrees of freedom (Dof), Kistler Force Platforms Type 9286AA) and kinematics, was performed using the SimiMotion System $^{\circledR}$ (Simi Reality Motion Systems GmbH, Unterschleißheim, Germany). A customized and bony landmark-based marker set of passive and reflective MoCap markers was used to record the motion of the lower extremities, pelvis and L4 (Figure 1(a)). Six digital cameras recorded the patient while walking on a $10 \mathrm{~m}$ walkway at a preferred walking speed in order to ensure comparability to other studies. ${ }^{29-33}$ The measured ground reaction forces and the trajectories of the MoCap markers retrieved from the gait analysis were used as the input for the musculoskeletal model to 


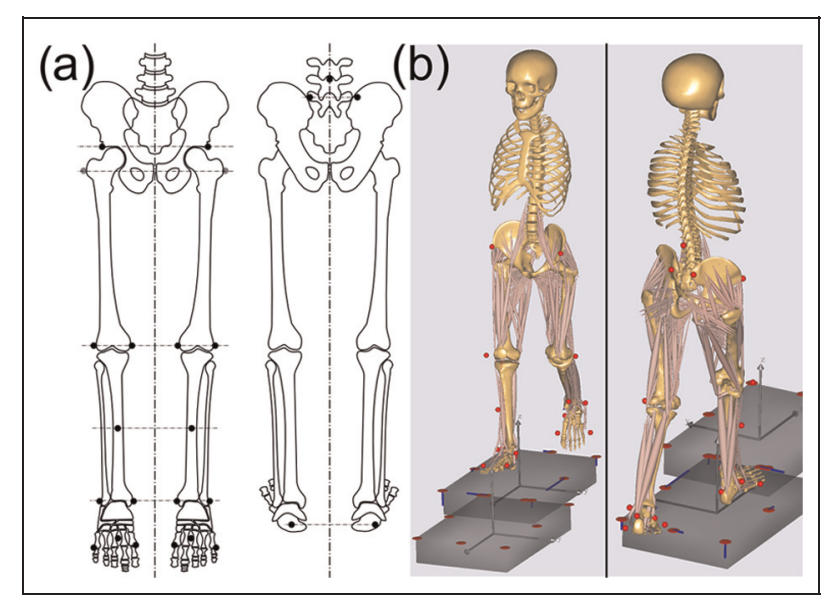

Figure I. (a) The specified marker set used in this study and its placement on the bony landmarks; (b) front and rear view of subject I during the musculoskeletal simulation.

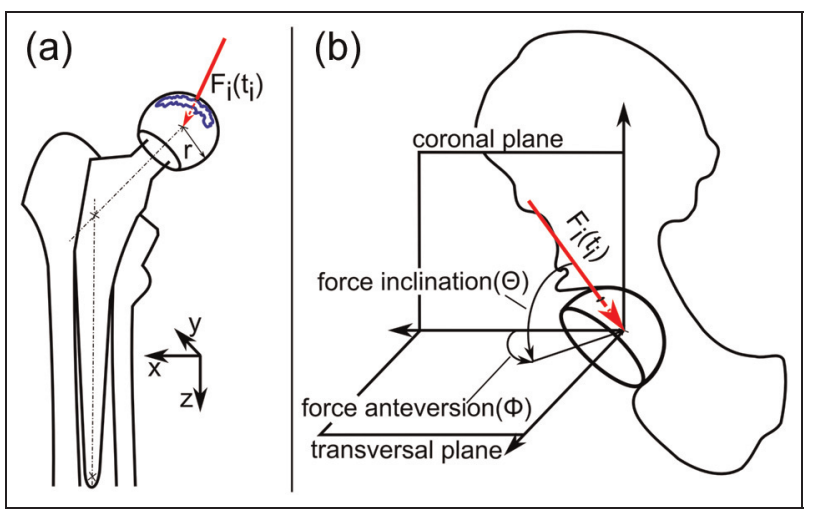

Figure 2. (a) Force path (line) on the head of the implant for every computed time step $\left(t_{i}\right)$ during the stance phase. (b) Force angles (and their definition) on the cup of the joint for every computed time step $\left(\mathrm{t}_{\mathrm{i}}\right)$ during the stance phase.

compute vectorial joint reaction forces for data processing. For the analyses the commercial software package AnyBody Modeling System (AnyBody Technology A/ $\mathrm{S}$, Aalborg, Denmark) has been utilized. The musculoskeletal model (AMMRV1.3.1) is scaled to the anthropometric properties of the patient using the fixed marker positions on the bony landmarks of the segments. Graphs and additional computations have been conducted by means of MATLAB R2010b Version 7.11.

To evaluate the orientation of the joint reaction forces on the head of the implant, the force path for the operated and non-operated joint has been calculated during the stance phase of the specific leg
(Figure 2). An example for a force path is shown in Figure 2. For every time step $\left(t_{i}\right)$ of the computation during the stance phase (one time step: 0.0137s) the point where the force vector $\left(\boldsymbol{F}_{\boldsymbol{i}}\right)$ meets the head of the implant has been computed, resulting in a force path for both joints. The individual diameter of the prosthesis head and the non-operated femoral head was taken into account. Measurements of the non-operated femoral head were retrieved from radiographs. For subject 1 this was $32 \mathrm{~mm}$ for the prosthesis head and $41 \mathrm{mmm}$ for the non-operated head size. For subject 2 the diameter of the prosthesis head was also $32 \mathrm{~mm}$ and $48 \mathrm{~mm}$ for the non-operated head size.

The vectorial joint reaction force in the femur reference coordinate system for every computed time step is (vectors are displayed in bold letters)

$$
\boldsymbol{F}_{\boldsymbol{i}}\left(t_{i}\right)=\left|F_{i}\left(t_{i}\right)\right| * \boldsymbol{e}_{\boldsymbol{i}}\left(t_{i}\right)
$$

A line is represented by

$$
\boldsymbol{l}: \boldsymbol{x}\left(t_{i}\right)=m\left(t_{i}\right) * \boldsymbol{D} \boldsymbol{V}_{\boldsymbol{i}}\left(t_{i}\right)+\boldsymbol{P} \boldsymbol{V}_{\boldsymbol{i}}\left(t_{i}\right)
$$

In this case the unit vector of the force is equal to the unit vector of the line and the position vector of the line is zero because it is assumed as a line through the origin

$$
\boldsymbol{D} \boldsymbol{V}_{\boldsymbol{i}}\left(t_{i}\right)=\boldsymbol{e}_{\boldsymbol{i}}\left(t_{i}\right) ; \boldsymbol{P} \boldsymbol{V}_{\boldsymbol{i}}\left(t_{i}\right)=0
$$

Therefore, derived from equation (2) and combined with equation (3) gives

$$
\boldsymbol{l}: \boldsymbol{x}\left(t_{i}\right)=m\left(t_{i}\right) * \boldsymbol{e}_{\boldsymbol{i}}\left(t_{i}\right)
$$

The points on the surface of a sphere in a general position in space are

$$
\boldsymbol{S}:\left(\boldsymbol{x}\left(t_{i}\right)-\boldsymbol{M}\right)^{2}=r^{2}
$$

where $\boldsymbol{M}$ is the given center of the sphere, $r$ is the given radius (scalar) and $\boldsymbol{x}$ is a point on the surface of the sphere. Based on equations (4) and (5), to calculate the points where the line meets the sphere, assumption (6) has to be satisfied

$$
\boldsymbol{l}=\boldsymbol{S}
$$

In order to satisfy equation (6) we need to solve equation (4) for the gradient $m$

$$
\left(m\left(t_{i}\right) * \boldsymbol{D} \boldsymbol{V}_{\boldsymbol{i}}-\boldsymbol{M}\right)^{2}=r^{2}
$$

Derived from equation (7) the equation for the gradient $m$ results in a quadratic equation for which two solutions exist. The non-possible solution was neglected and equation (7) results in

$$
m_{i}\left(t_{i}\right)=\frac{\boldsymbol{D} \boldsymbol{V}_{\boldsymbol{i}}\left(t_{i}\right) \cdot \boldsymbol{M}+\sqrt{\left(\boldsymbol{D} \boldsymbol{V}_{i}\left(t_{i}\right) \cdot \boldsymbol{M}\right)^{2}-\left(D V_{x, i}^{2}\left(t_{i}\right)+D V_{y, i}^{2}\left(t_{i}\right)+D V_{z, i}^{2}\left(t_{i}\right)\right)\left(M_{x}^{2}+M_{y}^{2}+M_{z}^{2}-r^{2}\right)}}{D V_{x, i}^{2}+D V_{y, i}^{2}+D V_{z, i}^{2}}
$$


where $D V_{x . i}, D V_{y . i}$ and $D V_{z . i}$ are the $x, y$ or $z$ component of the directional vector $\left(\boldsymbol{D} \boldsymbol{V}_{\boldsymbol{i}}\right)$ at every time step $t_{i}$, respectively.

By combining equation (8) with equation (4) the force path is computed

$$
\boldsymbol{P}_{\boldsymbol{i}}\left(t_{i}\right)=m_{i}\left(t_{i}\right) * \boldsymbol{D} \boldsymbol{V}_{\boldsymbol{i}}\left(t_{i}\right)
$$

To determine the orientation of the force vector on the cup of the prosthesis a similar technique like the force paths has been developed, aiming however, not for the position of the force vector on the cup, but for the inclination (or anteversion) angle of the force vector during the stance phase and for every computed hip reaction force at every time step $t_{i}$ with respect to the cup reference system. According to Figure 2(b), the force vector is transformed into a spherical coordinate system. The fixed radius of the spherical coordinate system ensures the comparability of the results, not only interindividual but also intra-individual. The use of a spherical coordinate system simplifies the computation of the force angles significantly and is calculated as following. Derived from equation (1) the conversion from Cartesian coordinates to spherical coordinates is conducted using equations (10) and (11) under the assumption $r=1$

$$
\begin{aligned}
& \theta=\tan ^{-1}\left(\frac{\boldsymbol{e}_{\boldsymbol{y}, \boldsymbol{i}}\left(t_{i}\right)}{\boldsymbol{e}_{\boldsymbol{x}, \boldsymbol{i}}\left(t_{i}\right)}\right) \\
& \boldsymbol{\phi}=\cos ^{-1}\left(\boldsymbol{e}_{z, i}\left(t_{i}\right)\right)
\end{aligned}
$$

The force angles are defined according to Murray ${ }^{34}$ and Lewinnek et al. ${ }^{40}$ (radiographic inclination (RI) and radiographic anteversion (RA)), where the force inclination angle is the angle between the force vector and the transversal plane $(\theta)$ and the force anteversion angle is the angle between the force vector and the coronal plane $(\phi)$.

\section{Results}

In Figure 3, the force pathways on the head of the implant of both hip joints during the stance phase for both subjects are displayed for the non-operated and operated side, respectively. All joint reaction forces have been normalized to the subject's specific bodyweight. The graph shows the force path in the transversal plane (anterior-posterior versus medio-lateral). The force path on the operated joint of subject 1 (inclination: $48.2^{\circ}$, anteversion: $19.6^{\circ}$ ) is located in the medial and posterior quarter. On the non-operated side, the force path is only located in the posterior-lateral quarter. The force path for subject 2 (inclination: $38.7^{\circ}$,

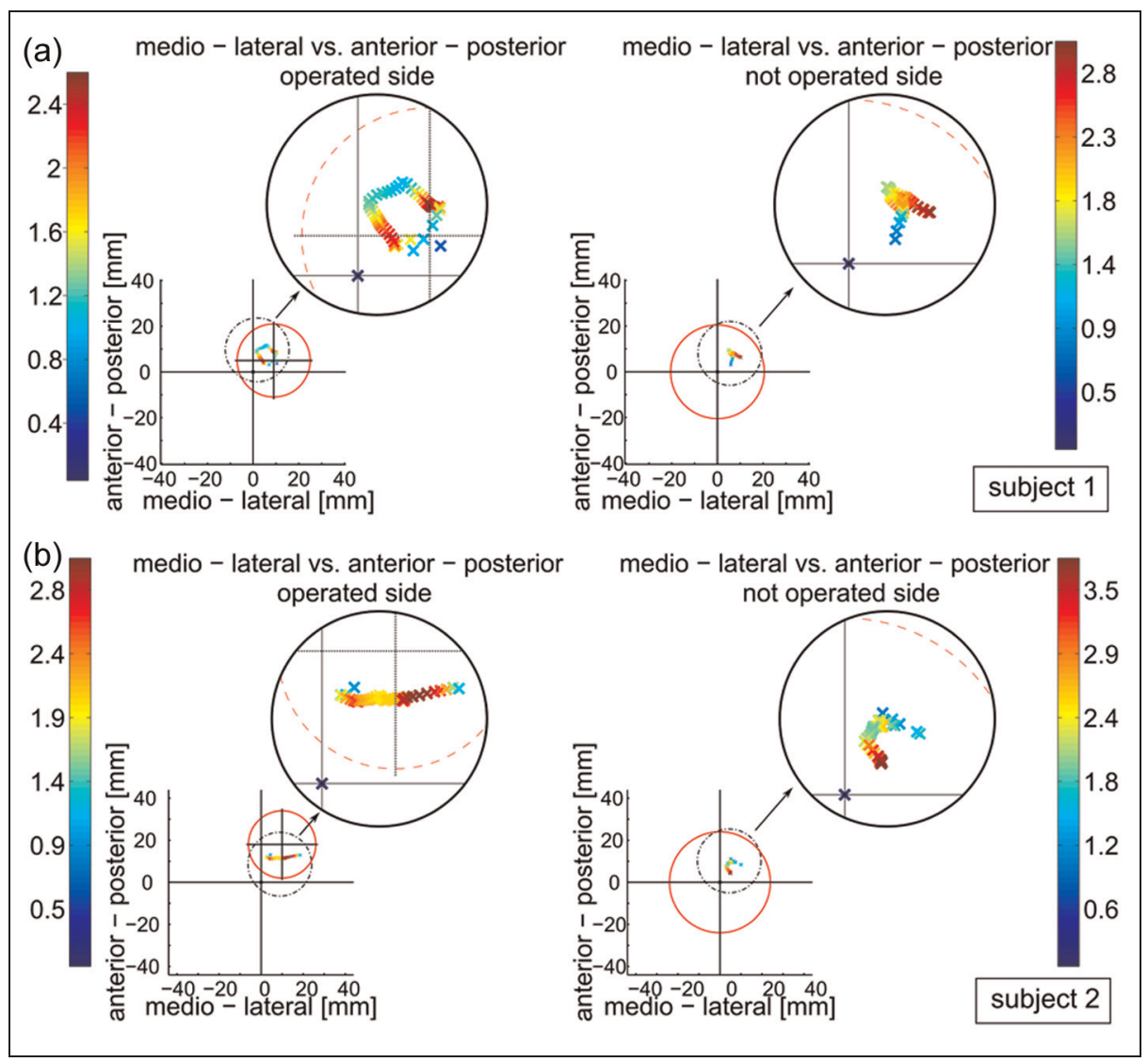

Figure 3. The post-operative force paths on the head of the implant on the operated hip joint (left) and on the non-operated hip joint (right) during the stance phase of subject I (a) and subject 2 (b) in the transversal plane. The prosthesis head is shown as a solid-drawn circle. To give a detailed insight, the force paths have been magnified, the dash-dotted circles represent the cut-out area. The shade of every point shows the magnitude of the hip reaction force with respect to the subjects maximum hip reaction force. 


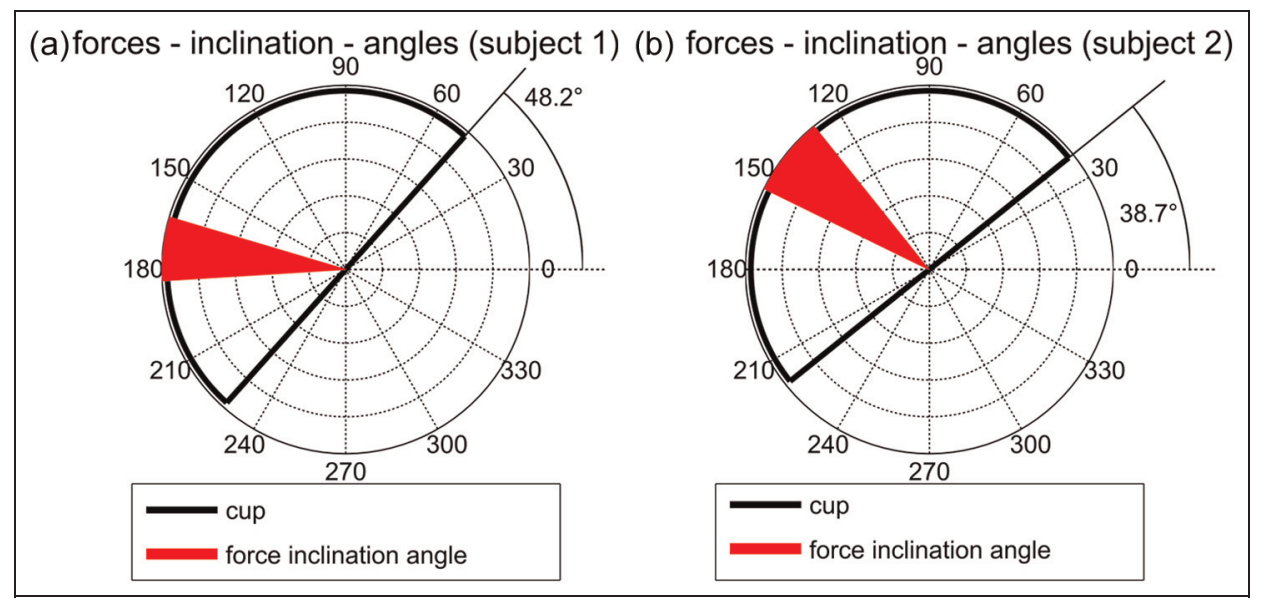

Figure 4. Inter-subject comparison of the force inclination angle on the cup during the stance phase; (a) subject I; (b) subject 2. The thick black line represents the cup orientation of the operated hip joint as it was measured post-operatively by CT-scans. The shaded area displays the force angles covered by the computed joint reaction force during stance.

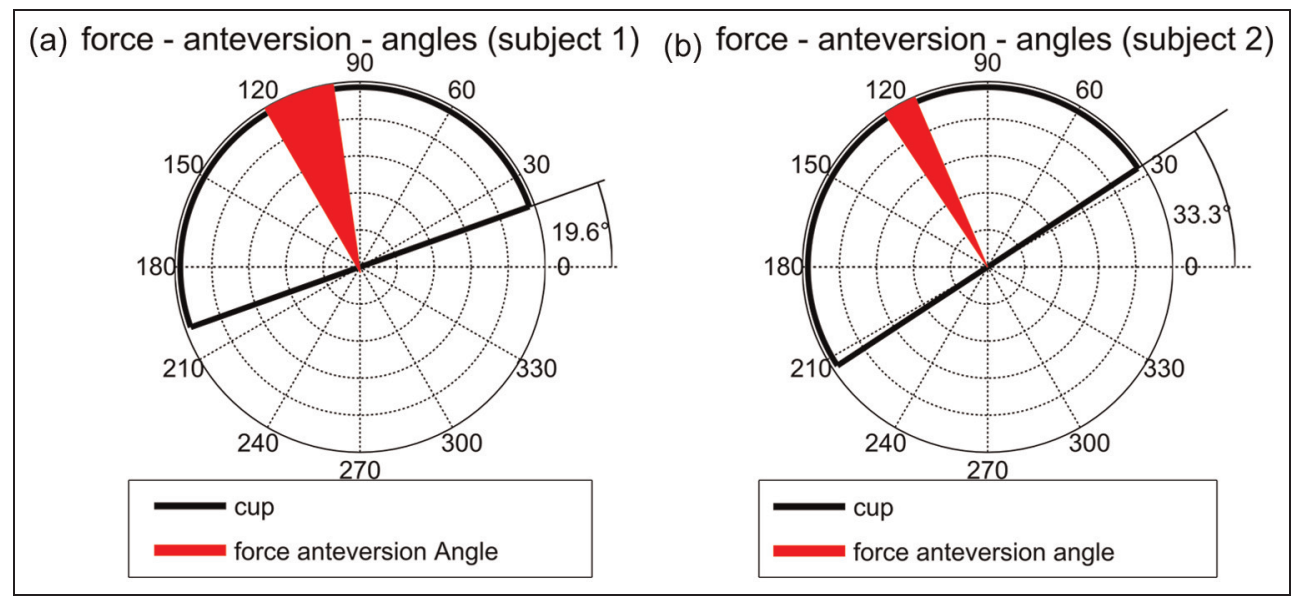

Figure 5. Inter-subject comparison of the force anteversion angle on the cup during the stance phase; (a) subject I; (b) subject 2. The thick black line represents the cup of the operated hip joint as it was measured post-operatively by CT-scans. The shaded area displays the force angles covered by the computed joint reaction force during stance.

anteversion: $33.3^{\circ}$ ) on the non-operated side is only located in the posterior-lateral quarter, as is the force path for subject 1 . In contrast to subject 1 the force path for subject 2, at the operated hip joint, can be found only within the anterior quarter of the head of the implant, where it is almost equally distributed in the medio-lateral half of the prosthesis head. The force paths during a complete stride cycle showed good agreement, in terms of shape, to the kinematic force tracks analysis. $^{24-26}$

Figure 4 shows a comparison of the force inclination angles between subject 1 and subject 2. As described before, subject 1 was picked because it showed the greatest cup inclination angle $\left(48.2^{\circ}\right.$ versus $\left.38.7^{\circ}\right)$ within a larger cohort. Also, the force inclination angle is greater compared with subject 2. However, the force inclination angles on the cup, with respect to the plane of the socket-face, indicates that the force vector is mainly positioned in the same quadrant when compared with subject 2 , in a cup-fixed coordinate system.
Figure 5 shows a comparison of the force anteversion angles between subject 1 and subject 2 . It can be observed that the covered region of the force anteversion angle is greater for subject 1. Also, the maximum force anteversion is greater for the subject with the greater anteversion angle, even if the effect is not as clear as for the inclination angle.

\section{Discussion}

The goal of our study was to show the feasibility of a novel method to analyze the hip reaction pathways and force angles after THR by means of post-operative gait analysis and including the development of subjectspecific musculoskeletal models. A qualitative comparison of different subjects, to evaluate the subject-specific magnitude, position and orientation of the hip reaction forces on the cup and stem, is presented to show that the subject-specific models indeed are capable of producing patient-specific results. 
The computed force paths and force angles indicate a subject-specific joint loading. With the exception of the operated hip joint in subject 2, all force paths are located within the posterior quadrant of the implant head. This is mainly owing to the fact that the subject's trunk leans forwards during the stance phase in order to conduct the swing phase of the opposite leg. Therefore, the hip joint has to provide a proper reaction force to keep the trunk above the hip. The force paths of both subjects on the non-operated hip joint are located in the lateral-posterior quarter of the implant head. However, the pathways differ if it comes to the operated side. The force path of subject 1 is mainly located in the medio-posterior quarter of the head. However, the force path for subject 2 can be found in the anterior half of the implant head, where it is nearly equally distributed in the medio-lateral half. In order to provide the necessary joint reaction force, the force in subject 2 is positioned more laterally than in subject 1 , because the subject had a greater step width in conjunction with a smaller pelvis width. We also hypothesize that this is owing to a protection mechanism. The patient simply pays more attention to prevent any overloading of the operated hip joint. This is confirmed by the greater joint reaction force on the non-operated side of the hip, which appears in both subjects on the same side (Figure 4).

The force inclination angle (Figure 5) also differs between the subjects. The region on the cup covered by the force inclination angle indicates that the orientation of the vectorial joint reaction force is highly patient-specific. Even if the force inclination angle differs between the subjects, its covered region on the cup (when looking at a cup-fixed coordinate system) is similar between the subjects, with respect to the plane of the socket face. The greater the inclination angle of the cup, the greater the force inclination angle. Therefore, the authors hypothesize that the individual orientation of the components with respect to each other ("combined anteversion") leads to a very similar load case on the cup during walking, ${ }^{36,37}$ while the absolute values of the force angles cover a wider range with respect to the hip or the stem. The force anteversion angle behaves similarly to the force inclination angle. Even when the effects are not as pronounced as seen the force inclination angle, one can see that the greater the anteversion angle, the greater the maximum-force anteversion angle becomes.

A strength of the proposed method is the skinmarker protocol used. The 27 markers that were applied on the bony landmarks of the body ensure that at least three markers were placed on every segment (the foot is modeled as one segment). To ensure a valid measurement of the hip-segment kinematics, owing to a high BMI (body mass index), the number of markers placed on the pelvis was also increased. The methods and models used in this study are taken from a public, open model repository (AMMR) and have been used and validated by numerous researchers. ${ }^{12-17,37,38}$ Also, the easy-to-use handling of over-determined musculoskeletal models during gait contributes to the modeling process. The open structure of the models provides the freedom to influence every parameter of the model. Of course, this might also be a possible source for errors, however, it gives us the opportunity to gain deep insight into the model - how it is developed and to modify its mechanical elements if necessary. One limitation of this study is the total number of two subjects, which is too little to state any quantitative declarations, yet enough to prove the feasibility of this pilot study. Also, the results determining the estimated region of wear have not yet been validated. This may be challenging, but can be done by comparing hip prosthesis that has been worn by patients for many years with the computed regions of wear. One might also use a hip joint simulator to compare the computed regions of wear, however, comparing them to an actually worn prosthesis is the first step towards a proper validation of this technique. Another caveat of this study is the strong scattering of the post-operative time of the gait analysis. The influence of this parameter can, therefore, not be determined and will be a focus of a future study. ${ }^{20}$

The proposed methods in this article are aimed at defining two functional outcomes of THR that are related to wear and rim loading. One important cause for the long term failure of a hip prosthesis is the excessive accumulation of wear debris, ${ }^{39,40}$ which can induce an osteolytic reaction, leading to component loosening and implant failure. ${ }^{41}$ It is accepted that wear is not only a function of time but a function of use, ${ }^{42}$ and dependent on the material properties (wear factor), the force applied to the prosthesis and the sliding distance of the cup with respect to the prosthesis head. ${ }^{43,44}$ Calonius and Saikko ${ }^{24,26}$ and Saikko and Calonius ${ }^{25}$ developed a technique to measure the motion between a cup and the prosthesis head, respectively, and succeeded in computing these slide tracks using finite element analysis. However, the kinematics during the gait cycle not only influences the wear rates but also the applied forces, ${ }^{43,44}$ which, of course, are highly subjectspecific and dependent on the multidirectional motion that occurs during gait. ${ }^{45,46}$ Owing to the methods listed in this article we are able to compute (a) the applied force and (b) the sliding distance (force pathway) for every subject, respectively. Future work on a properly defined patient collective will be employed in order to answer quantitative questions. Concerning critical subject-specific hip joint loading (rim loading) on the cup after THR, only a few biomechanical studies have been conducted either by means of a test rig ${ }^{21,22}$ or a computational model. ${ }^{22,23}$ Their results indicate a biomechanical mechanism dealing with the resisting moment in a hip joint, which is mainly dependent on the distance of the resulting joint reaction force to the rim of the cup. The computed hip reaction force angles and the distance to the rim cup are a measurement for cup or rim loading and whether it occurs in the socalled safe-zones. $^{35}$ 
Nadzadi et al. ${ }^{27}$ analyzed different risk movement patterns by means of MoCap data performed on a healthy subject, and a computational model. They propose that shearing and sliding-out processes between the stem and the cup might be involved in the failure of hip implants. The multidirectional motion of the force pathways and a correlation to the resisting moment, ${ }^{23}$ retrieved from the force angles, may provide a new understanding of hip joint failure mechanisms, once quantified. In particular, the vectorial pathways and the orientation of the hip reaction forces seem to offer a promising approach to clarify these effects, especially once the focus of these analyzing techniques lie, not only on the (as safe movement considered) gait of the patients, but also on movements such as sit-to-stand or stairclimbing. The protocol of a future study includes a knee bend motion that servers as an approach for those critical motions. ${ }^{20}$ It also includes a corresponding preoperative gait analysis, by which means one may be able to determine a possible relationship between pre- and post-operative wear rates. This may open the door to be one day able to predict occurring wear rates by means of a pre-operative gait analysis. ${ }^{44}$ However, the goal of this study is to determine the feasibility of a method to define these two functional outcomes following THR. Not only can these measurements be used as a functional outcome following THR, but also to enhance the general understanding of the hip biomechanics after THR.

Combining gait analysis with musculoskeletal modeling is valid and meaningful to research into the functional outcome after receiving THR. This article proposes two definitions for the functional outcome by means of objective measurements, such as gait analysis and musculoskeletal models. This method may well give us the insight into the biomechanical situation during gait after receiving THR that we need to fully understand the mechanisms acting on a hip joint and to prove a possible increase of functional outcome after receiving THR.

\section{Funding}

This research received no specific grant from any funding agency in the public, commercial, or not-for-profit sectors.

\section{References}

1. Renkawitz T, Schuster T, Herold T, et al. Measuring leg length and offset with an imageless navigation system during total hip arthroplasty: is it really accurate? Int $J$ Med Robot 2009; 5(2): 192-197.

2. van den Bogert A, Read L and Nigg BM. A method for inverse dynamic analysis using accelerometry. $J$ Biomech 1996; 29(7): 949-954.

3. Boardman DL, Dorey F, Thomas BJ, et al. The accuracy of assessing total hip arthroplasty outcomes: a prospective correlation study of walking ability and 2 validated measurement devices. J Arthroplasty 2000; 15(2): 200-204.

4. Long WT, Dorr LD, Healy B, et al. Functional recovery of noncemented total hip arthroplasty. Clin Orthop Relat Res 1993; (288): 73-77.
5. Hulet C, Hurwitz DE, Andriacchi TP, et al. Functional gait adaptations in patients with painful hip. Rev Chir Orthop Reparatrice Appar Mot 2000; 86(6): 581-589.

6. Bhargava P, Shrivastava P and Nagariya Sp. Assessment of changes in gait parameters and vertical ground reaction forces after total hip arthroplasty. Indian J Orthop 2007; 41(2): 158-162.

7. vanden Akker-Scheek I, Stevens M, Bulstra S, et al. Recovery of gait after short-stay total hip arthroplasty. Arch Phys Med Rehabil 2007; 88(3): 361-367.

8. Foucher KC, Wimmer MA, Moisio KC, et al. Time course and extent of functional recovery during the first postoperative year after minimally invasive total hip arthroplasty with two different surgical approaches - a randomized controlled trial. J Biomech 2011; 44(3): 372-378.

9. Bergmann G, Graichen F and Rohlmann A. Hip joint loading during walking and running, measured in two patients. J Biomech 1993; 26(8): 969-990.

10. Bergmann G, Deuretzbacher G, Heller M, et al. Hip contact forces and gait patterns from routine activities. $J$ Biomech 2001; 34(7): 859-871.

11. Heller M, Bergmann G, Deuretzbacher G, et al. Musculo-skeletal loading conditions at the hip during walking and stair climbing. J Biomech 2001; 34(7): 883-893.

12. Pandy MG. Computer modeling and simulation of human movement. Annu Rev Biomed Eng 2001; 3: 245-273.

13. Erdemir A, McLean S, Herzog W, et al. Model-based estimation of muscle forces exerted during movements. Clin Biomech (Bristol, Avon) 2007; 22(2): 131-154.

14. Lenaerts G, De Groote F, Demeulenaere B, et al. Subject-specific hip geometry affects predicted hip joint contact forces during gait. $J$ Biomech 2008; 41(6): 1243-1252.

15. Jonkers I, Sauwen N, Lenaerts G, et al. Relation between subject-specific hip joint loading, stress distribution in the proximal femur and bone mineral density changes after total hip replacement. J Biomech 2008; 41(16): 3405-3413.

16. de Zee M, Dalstra M, Cattaneo PM, et al. Validation of a musculo-skeletal model of the mandible and its application to mandibular distraction osteogenesis. $J$ Biomech 2007; 40(6): 1192-1201.

17. de Zee M and Rasmussen J. Using musculoskeletal modeling for estimating the most important muscular output: force. In: Proceedings of the 3D physiological human workshop, 3DPH, Zermatt, Switzerland, Lecture Notes in Computer Science 5903. Springer, 2009.

18. Andersen MS. Kinematically over-determinate musculoskelatal systems. PhD Thesis, AAlborg University, 2009.

19. Damsgaard M, Rasmussen J, Tørholm S, et al. Analysis of musculoskeletal systems in the anybody modeling system. Simulation Modell Practice Theory 2006; 14: $1100-1111$

20. Renkawitz T, Haimerl M, Dohmen L, et al. Minimally invasive computer-navigated total hip arthroplasty, following the concept of femur first and combined anteversion: design of a blinded randomized controlled trial. BMC Musculoskelet Disord 2011; 12: 192.

21. Nicholas RM, Orr JF, Mollan RA, et al. Dislocation of total hip replacements. a comparative study of standard, long posterior wall and augmented acetabular components. J Bone Joint Surg Br 1990; 72(3): 418-422.

22. Scifert CF, Brown TD, Pedersen DR, et al. A finite element analysis of factors influencing total hip dislocation. Clin Orthop Relat Res 1998; 355: 152-162. 
23. Stewart KJ, Pedersen DR, Callaghan JJ, et al. Implementing capsule representation in a total hip dislocation finite element model. Iowa Orthop J 2004; 24: 1-8.

24. Calonius O and Saikko V. Slide track analysis of eight contemporary hip simulator designs. J Biomech 2002; 35(11): 1439-1450.

25. Saikko V and Calonius O. Slide track analysis of the relative motion between femoral head and acetabular cup in walking and in hip simulators. J Biomech 2002; 35(4): 455-464.

26. Calonius $\mathrm{O}$ and Saikko V. Force track analysis of contemporary hip simulators. J Biomech 2003; 36(11): 1719-1726.

27. Nadzadi ME, Pedersen DR, Yack HJ, et al. Kinematics, kinetics, and finite element analysis of commonplace maneuvers at risk for total hip dislocation. $J$ Biomech 2003; 36(4): 577-591.

28. Murray MP, Gore DR, Brewer BJ, et al. A comparison of the funtional performance of patients with charnley and müller total hip replacement. a two-year follow-up of eighty-nine cases. Acta Orthop Scand 1979; 50(5): 563-569.

29. Berman AT, Quinn RH and Zarro VJ. Quantitative gait analysis in unilateral and bilateral total hip replacements. Arch Phys Med Rehabil 1991; 72(3): 190-194.

30. Loizeau J, Allard P, Duhaime M, et al. Bilateral gait patterns in subjects fitted with a total hip prosthesis. Arch Phys Med Rehabil 1995; 76(6): 552-557.

31. Perron M, Malouin F, Moffet H, et al. Three-dimensional gait analysis in women with a total hip arthroplasty. Clin Biomech (Bristol, Avon) 2000; 15(7): 504-515.

32. Madsen MS, Ritter MA, Morris HH, et al. The effect of total hip arthroplasty surgical approach on gait. J Orthop Res 2004; 22(1): 44-50.

33. Miki H, Sugano N, Hagio K, et al. Recovery of walking speed and symmetrical movement of the pelvis and lower extremity joints after unilateral THA. J Biomech 2004; 37(4): 443-455.

34. Murray DW. The definition and measurement of acetabular orientation. J Bone Joint Surg Br 1993; 75(2): 228-232.

35. Lewinnek GE, Lewis JL, Tarr R, et al. Dislocations after total hip-replacement arthroplasties. J Bone Joint Surg Am 1978; 60(2): 217-220.

36. D'Lima DD, Urquhart AG, Buehler KO, et al. The effect of the orientation of the acetabular and femoral components on the range of motion of the hip at different head-neck ratios. J Bone Joint Surg Am 2000; 82(3): 315-321.

37. Heller M, Bergmann G, Deuretzbacher G, et al. Influence of femoral anteversion on proximal femoral loading: measurement and simulation in four patients. Clin Biomech (Bristol, Avon) 2001; 16(8): 644-649.

38. Dubowsky SR, Rasmussen J, Sisto SA, et al. Validation of a musculoskeletal model of wheelchair propulsion and its application to minimizing shoulder joint forces. $\mathrm{J}$ Biomech 2008; 41(14): 2981-2988.

39. Willert HG and Semlitsch M. Reactions of the articular capsule to wear products of artificial joint prostheses. J Biomed Mater Res 1977; 11(2): 157-164.

40. Ingham $\mathrm{E}$ and Fisher $\mathrm{J}$. The role of macrophages in osteolysis of total joint replacement. Biomaterials 2005; 26(11): 1271-1286.
41. Schmalzried TP, Jasty M and Harris WH. Periprosthetic bone loss in total hip arthroplasty. polyethylene wear debris and the concept of the effective joint space. $J$ Bone Joint Surg Am 1992; 74(6): 849-863.

42. Schmalzried TP, Shepherd EF, Dorey FJ, et al. The john charnley award. wear is a function of use, not time. Clin Orthop Relat Res 2000; 381: 36-46.

43. Maxian TA, Brown TD, Pedersen DR, et al. A slidingdistance-coupled finite element formulation for polyethylene wear in total hip arthroplasty. J Biomech 1996; 29(5): 687-692.

44. Kang L, Galvin AL, Brown TD, et al. Wear simulation of ultra-high molecular weight polyethylene hip implants by incorporating the effects of cross-shear and contact pressure. Proc IMechE, Part H: J Engineering in Medicine 2008; 222(7): 1049-1064.

45. Bragdon CR, O'Connor DO, Lowenstein JD, et al. The importance of multidirectional motion on the wear of polyethylene. Proc IMechE, Part H: J Engineering in Medicine 1996; 210(3): 157-165.

46. Bennett D, Humphreys L, O'Brien S, et al. Wear paths produced by individual hip-replacement patients-a largescale, long-term follow-up study. J Biomech 2008; 41(11): 2474-2482.

\section{Appendix}

\section{Notations}

$\boldsymbol{e}_{\boldsymbol{i}}\left(t_{i}\right) \quad$ directional vector of the force at

designated time step $t_{i}$

$\boldsymbol{l} \quad$ common representation of a line in general position in space

$m\left(t_{i}\right) \quad$ gradient $m$ at designated time step $t_{i}$ of the line passing through the force vector patient-specific hip joint radius of either operated (prosthesis head) or nonoperated hip joint (femoral head retrieved from radiographs)

$t_{i} \quad$ designated time step of the computation where $i=1, \ldots, n$ and one time step is $0.0137 \mathrm{~s}$

$\boldsymbol{x}\left(t_{i}\right) \quad$ points on a line in general position in space at the designated time step

$\boldsymbol{D} \boldsymbol{V}_{\boldsymbol{i}}\left(t_{i}\right) \quad$ directional vector of the line in general position in space at the designated time step

$\boldsymbol{F}_{i} \quad$ force vector at the designated time step retrieved from the musculoskeletal model center of the implant head in the vectorial form $M_{x}, M_{y}, M_{z}$ as retrieved by the CAS equipment

$\boldsymbol{P}_{i}\left(t_{i}\right) \quad$ force path point on the prosthesis head with the radius $r$ at the designated time step

$\boldsymbol{P} \boldsymbol{V}_{\boldsymbol{i}}\left(t_{i}\right) \quad$ position vector of a line in general space

$\mathbf{S}$ common representation of points on a sphere in general position in space

$\theta \quad$ force inclination angle

$\phi \quad$ force anteversion angle 